\title{
Indagini multidisciplinari ad Aquinum: la valorizzazione delle conoscenze
}

Giuseppe Ceraudo

Università del Salento

email: giuseppe.ceraudo@unisalento.it

\section{Abstract}

The urban area of Aquinum, a Roman city situated along via Latina between Frosinone (Frusino) and Cassino (Casinum), was investigated by aero-topographical st udies, geophysical prospecting and archaeological field survey. All research activity was done by the Laboratory of Ancient Topography and Photogrammentry of the University of Salento. The executed explorative interventions have considered the results of the data processed as well as all documents related to the systematic surveys realised in the framework of the Ager Aquinas project. The substance of the archaeological deposits, the wealth of ancient materials and the remains highlighted constitute indicators of positive conditions for the planning of archaeological research oriented to confirming all that was individuated by the field surveys, aerial photographs and geophysical prospecting. These studies have allowed the reconstruction of the urban layout of Aquinum. Planning for the archaeological interventions was based not only on the results but also on the processing of data regarding field survey and on the compelling need for the preservation of the area.

Starting with the collected data, six archaeological excavation campaigns have been carried out between 2009 and 2014 in Aquinum. The research, after confirming the aerotopographical and geophysical results, focused on an area of the town that, until that time, had provided nothing useful for the recognition of monuments and urban spaces. Great results have been obtained thanks to the archaeological excavation campaigns led at the heart of the ancient Roman town. Knowledge of a central part of the built-up area has improved including where there are public buildings already known (theatre, an apsis-building) and unknown (public thermal baths). This is the keystone for better protection of the archaeological site and to future preservation for the area.

Keywords: Aquinum, Aero-Topographical Studies, Geophysical Prospecting and Archaeological Field Survey, Public Thermal Baths

\section{Il Progetto Ager Aquinas}

Tra le attività di ricerca portate avanti dal Laboratorio di Topografia Antica e Fotogrammetria (LabTAF) dell'Università del Salento si inserisce il Progetto Ager Aquinas, avviato, ormai da alcuni anni, con il sostegno dei Comuni di Aquino e di Castrocielo ed in collaborazione con la Soprintendenza per i Beni Archeologici del Lazio (Ceraudo, 2012: 94-104). Il progetto, avviato nel 1999 con campagne sistematiche di ricognizione topografica, grazie anche al supporto dell'allora amministrazione comunale di Aquino, dopo dieci anni di indagini topografiche, aerofotografiche, geognostiche, attraverso le quali è stata redatta una prima fotogrammetria finalizzata, è finalmente approdato allo scavo archeologico, grazie all'interessamento del comune di Castrocielo, che ha messo a disposizione del progetto di ricerca una vasta area di proprietà, sita in un settore nevralgico della città antica (Ceraudo \& Murro, 2014).

L'attività sul campo e di laboratorio, è stata condotta seguendo quelle linee di ricerca che da sempre caratterizzano la ricerca topografica del Laboratorio leccese: esplorazione archeologica del territorio mediante ricognizione diretta sistematica, fotogrammetria finalizzata all'archeologia, 
uso e applicazioni specialistiche della fotografia aerea verticale ed obliqua, rilievo e analisi dei monumenti e, più di recente, ricognizioni aeree a bassa quota e prospezioni geofisiche.

Due le principali particolarità della città romana di Aquinum: la prima è costituita dal sistema difensivo formato da una cinta muraria e da un largo fossato; il profondo taglio difendeva tre dei quattro lati del perimetro urbano, mentre il quarto lato, ad Est, era difeso naturalmente dalla presenza di tre laghi prosciugati e bonificati verso la fine del XVI secolo; la seconda caratteristica è dovuta alla disposizione regolare ma non ortogonale degli assi del reticolo stradale cittadino, la cui inconsueta disposizione dava origine ad isolati a forma di parallelogramma (fig. 1) (Ceraudo, 2004: 13-24, fig. 18).

Le caratteristiche del perimetro e della rete stradale dell'abitato sono stati ricostruiti grazie alla fotointerpretazione di immagini aeree storiche. Dallo studio di foto della Royal Air Force britannica scattate nel febbraio del 1944, analizzate dal Giuliani (1964: 41-49), scaturirono le prime fondate acquisizioni sull'urbanistica della città, sulle dimensioni di massima degli isolati, sulla natura del fossato difensivo.

Si è potuto così ricostruire lo schema urbano, regolare ma con assi stradali che non si incrociavano ad angolo retto, a partire proprio dai due assi generatori: la via Latina che attraversava in linea retta Aquinum da ovest ad est, e ne costituiva l'asse stradale principale (il cd. Decumanus Maximus) e la via Montana, quella strada che doveva avere grossomodo l'andamento dell'attuale via Vicinale Civita Vetere (il cosiddetto Cardo Maximus Maximus). La mancanza di requisiti fotogrammetrici delle foto, però, non consentì di arrivare a definire, se non orientativamente, le esatte dimensioni degli isolati: la struttura modulare dell'impianto urbano di Aquinum è stata così solo di recente calcolata, ricostruendo isolati maggiori di 212 × 140 m $16 \times 4$ actus), ulteriormente divisi in fasce più strette di 2 actus $(70 \mathrm{~m})$, con gli ultimi due settori nella parte settentrionale della città di 6 x 4,5 actus. In questo settore il modulo risulta leggermente sfalsato rispetto al sistema generale, perché probabilmente condizionato dalla presenza di un grande Tempio, il cd. Capitolium, e quindi di un'area sacra nell'angolo nord-occidentale dell'area urbana (Ceraudo, 1999: 161-68).

La realizzazione dell'aerofotogrammetria, direttamente restituita da un archeologo, ha permesso di acquisire una conoscenza complessiva e di dettaglio delle caratteristiche morfologiche della zona, ed ha consentito di restituire nella cartografia di base tutte quelle tracce desunte da numerosi voli di epoche diverse recuperati presso diversi archivi aerofotografici pubblici e privati.

Grande spazio è stato dedicato all'analisi specialistica della fotografia aerea verticale, strumento fondamentale per la conoscenza e la documentazione negli studi di “topografia archeologica”, che con le sue applicazioni rappresenta una delle fonti che ha offerto la maggior quantità di risultati utili per gli studi del settore.

La linea delle mura risulta coerente con la rete stradale urbana e secondo questa orientata; sul lato orientale, come già evidenziato, la fortificazione doveva seguire l'andamento del ciglio del Vallone di Aquino. In questo caso, tutto il vasto settore ad Est a ridosso dei laghi, poi interessato dal primo insediamento medievale di Aquino, è da inserire all'interno dell'area urbana e diviene parte integrante della città antica, una sorta di acropoli che si affacciava sui laghi e dominava da una posizione favorevole l'intera estensione cittadina. Con questa sostanziale variazione la superficie dell'abitato risulta essere di poco superiore ai 100 ettari rispetto agli 85 prima stimati (Ceraudo, 1999: 161-68); sicuramente la città più grande in età tardo-repubblicana e imperiale in questo nevralgico comparto territoriale al confine tra Lazio e Campania, posto sulla linea di collegamento tra Roma e Capua, alternativa interna al passaggio lungo la costa tirrenica della via Appia.

I risultati appena riassunti luna sintesi in Ceraudo, 2004), possono essere considerati i più importanti raggiunti nel corso delle prime indagini aerotopografiche effettuate sul sito, inoltre, possono essere considerati il punto di partenza per le nuove indagini che in questi ultimi anni hanno interessato un'area nevralgica all'interno dell'area urbana. 


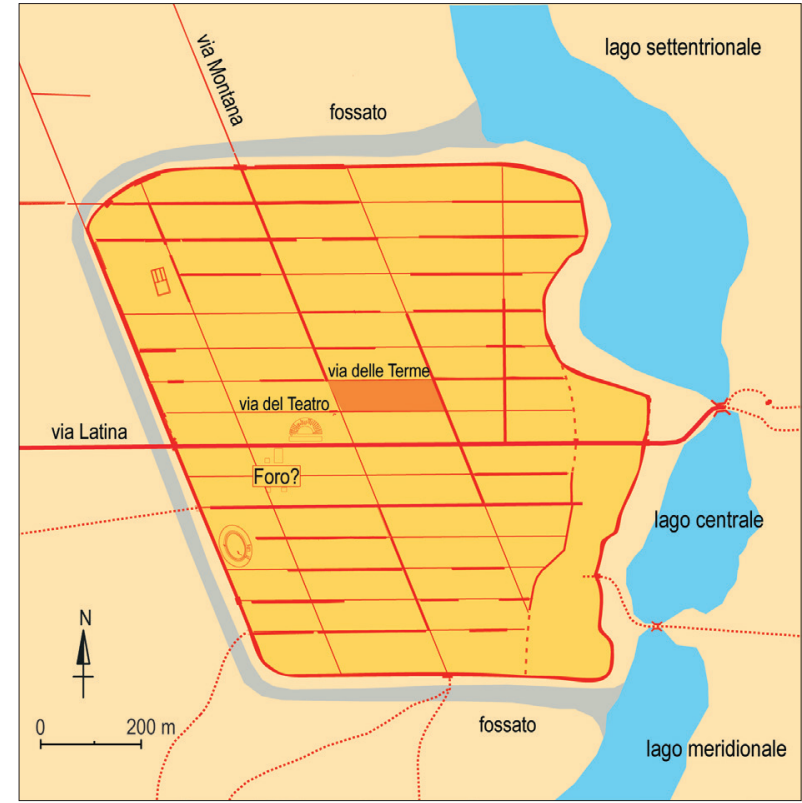

Fig. 1. Lo schema urbanistico di Aquinum, evidenziato in arancio l'isolato interessato dall'edificio termale.

\section{Le nuove ricerche (2008-2014)}

Il settore oggetto della ricerca, di proprietà del Comune di Castrocielo, è localizzato a nord del tracciato dell'Autostrada del Sole "A1" ed è disposto su una superficie pianeggiante estesa per quasi 8 ettari. L'area si trova all'interno del perimetro urbano della città romana, è collocato subito a nord dell'antica via Latina e ad est del teatro e del c.d. edificio absidato, dai quali, in antico, era separato dal Cardo Maximus, denominato via Montana (v. fig. 1).

\section{Le indagini aero-topografiche}

Dal 2008, grazie all'avvio di uno specifico programma di ricognizione aerea del territorio, con decine e decine di voli mirati effettuati in periodi differenti dell'anno, è stato possibile riconoscere in traccia i resti di edifici pubblici e privati in alcuni settori centrali dell'abitato e di definire, con maggiore precisione, il tracciato di alcuni assi viari, sia all'interno della maglia urbana sia all'esterno della linea delle mura (Ceraudo, 2012: 94-103).

\section{Ricognizione topografica}

Per quanto riguarda l'attività sul campo, con l'intento di rinnovare questo filone di ricerca, pur senza perdere di vista gli obiettivi fondamentali di conoscenza da raggiungere attraverso l'indagine topografica sul territorio, va ribadito che questo tipo di lavoro viene necessariamente supportato dalle consolidate tecniche di ricognizione diretta sul terreno. Infatti, uno degli elementi che più qualifica la topografia d'indirizzo archeologico è proprio la ricognizione sul campo, integrale e sistematica. Ai risultati delle ricognizioni sono stati applicati nuovi metodi di registrazione cartografica, delle presenze e della distribuzione delle testimonianze archeologiche, tenendo conto anche delle assenze e dei fattori geografico-ambientali che possono aver influenzato, condizionato, limitato o impedito la lettura diretta sul terreno, descrivendone il grado di leggibilità in carte delle “visibilità" del terreno ricognito.

Attraverso le indagini sul terreno sono state individuate delle aree con una notevole concentrazione di materiale archeologico; in seguito alle riprese aeree, in cui erano nitidamente visibili tracce di strade e di strutture, è stato possibile mettere in relazione tali aree di frammenti con edifici isolati (verosimilmente delle domus) allineati lungo gli assi stradali.

\section{Prospezioni geofisiche}

L'opportunità di sperimentare strumenti innovativi e nuove metodologie di indagine deriva proprio dalla lunga esperienza condotta con i metodi appena descritti, dalla consapevolezza dei limiti ad essi connaturati e dalla constatazione del progressivo depauperamento delle evidenze di superficie. Per questo motivo è stata avviata una prima campagna di prospezioni georadar e magnetometriche con l'obiettivo di testare le potenzialità di questo tipo 
d'indagine integrando ed incrociando i dati già in nostro possesso, con il proposito di ripetere progressivamente l'esperienza su più ampia scala in tutti i terreni all'interno del perimetro urbano. Le prospezioni effettuate hanno coperto per ora una superficie totale di circa 10 ha, le griglie sono state localizzate e georeferenziate in un settore che, come abbiamo visto, era ricco di materiale archeologico di superficie e di tracce archeologiche. Anche in questo caso i risultati conseguiti sono stati entusiasmanti, confermando pienamente i dati ottenuti con il lavoro di ricognizione e di fotointerpretazione archeologica ed evidenziando ancor meglio la localizzazione delle strutture sepolte lle prospezioni sono state effettuate in collaborazione con colleghi del Laboratorio di Archeologia dei Paesaggi dell'Università di Siena diretto da S. Campana e dal CNR ITABC sotto la direzione dei S. Piro).

\section{Scavo archeologico}

A partire dai dati acquisiti sul campo, grazie ancora al contributo del Comune di Castrocielo, dal 2009 al 2014 ad Aquinum sono state condotte sei campagne di scavo archeologico che hanno permesso di riportare alla luce le strutture di un edificio termale di estremo interesse per dimensioni, ricchezza decorativa e articolazione degli ambienti (fig. 2). L'avanzamento degli studi sul sito ha portato finora a definire in maniera puntuale planimetria e funzione degli spazi (fig. 3). Le Terme Centrali, così come sono state definite, sono del tipo cosiddetto "pompeiano", ossia interamente inserite all'interno di un isolato, delimitato a nord e a sud da due grandi decumani basolati paralleli a $\mathrm{N}$ alla via Latina, convenzionalmente chiamati "Via delle Terme" e "Via del Teatro". Le strutture sono disposte secondo un doppio orientamento lest-ovest e nord ovest-sud est) coerente con la viabilità urbana di Aquinum (Ceraudo, 1999: 161-68; da ultimo Ceraudo, 2012: 94-103) (cfr. fig. 1). La presenza di orientamenti duplici, che ritroviamo anche in complessi pompeiani ed ostiensi, se da un lato evidenzia una organizzazione rigida dei vani subordinata ad un impianto stradale preesistente, dall'altro permette di comprendere meglio l'organizzazione spaziale delle opere di ampliamento, evidenti nel settore est dell'area di scavo, opere che hanno inoltre portato ad una rifunzionalizzazione ed a modifiche planimetriche di alcuni ambienti.

Risultano ad oggi scavati oltre 40 vani, distribuiti su una superficie di circa $5.000 \mathrm{mq}$, che tuttavia costituiscono ancora soltanto una parte dell'intero monumento, che ha il suo limite certo unicamente sul lato settentrionale, con una fronte stradale di oltre $80 \mathrm{~m}$ (fig. 4). Al momento sono ancora da definire i limiti lungo i lati orientale e occidentale. Molto probabilmente il decumano rinvenuto in prossimità del Casale Pascale, sempre all'interno dell'Area archeologica, sembrerebbe costituire il limite meridionale dell'intero complesso (v. fig. 3).

L'ampliamento dell'area di scavo verso 0 ha portato al riconoscimento di un ambiente che presenta una serie di pilastri in laterizio ancora parzialmente intonacati (probabili resti di un portico che sembrerebbe essere l'ingresso principale sul quel lato), e farebbe riferimento ad una nuova fase di regolarizzazione edilizia del lato occidentale dell'edificio pubblico. Gli accessi alle terme finora individuati con certezza sono tre: uno meridionale, al quale si accedeva da una strada in basoli scuri di certo collegata alla via Latina, e due nel settore $\mathrm{N}$, lungo la monumentale Via delle Terme. La presenza di due grandi frigidaria in corrispondenza dei due ingressi sul lato settentrionale, uno nel blocco orientale ed uno in quello occidentale, rende assai probabile l'ipotesi di un utilizzo simultaneo del complesso da parte di uomini e donne. Nel frigidarium orientale, merita particolare attenzione la presenza dell'iscrizione musiva collocata all'interno di una tabula ansata con l'attestazione di due notabili locali: Plotius Albanus e Mevius Festus. Entrambi i personaggi (ma forse ne potevano essere nominati altri due nella parte purtroppo distruttal devono esser legati all'ambiente/edificio connesso al mosaico. Forse magistrati promotori o restauratori di un ambiente. Il mosaico, dal punto di vista tipologico, è inquadrabile nel I sec. d.C. (Ceraudo, Molle \& Nonnis, 2013: 101-10, in part. 102-106, con bibl.).

L'assetto planimetrico delle terme appare decisamente determinato da preesistenze stradali 


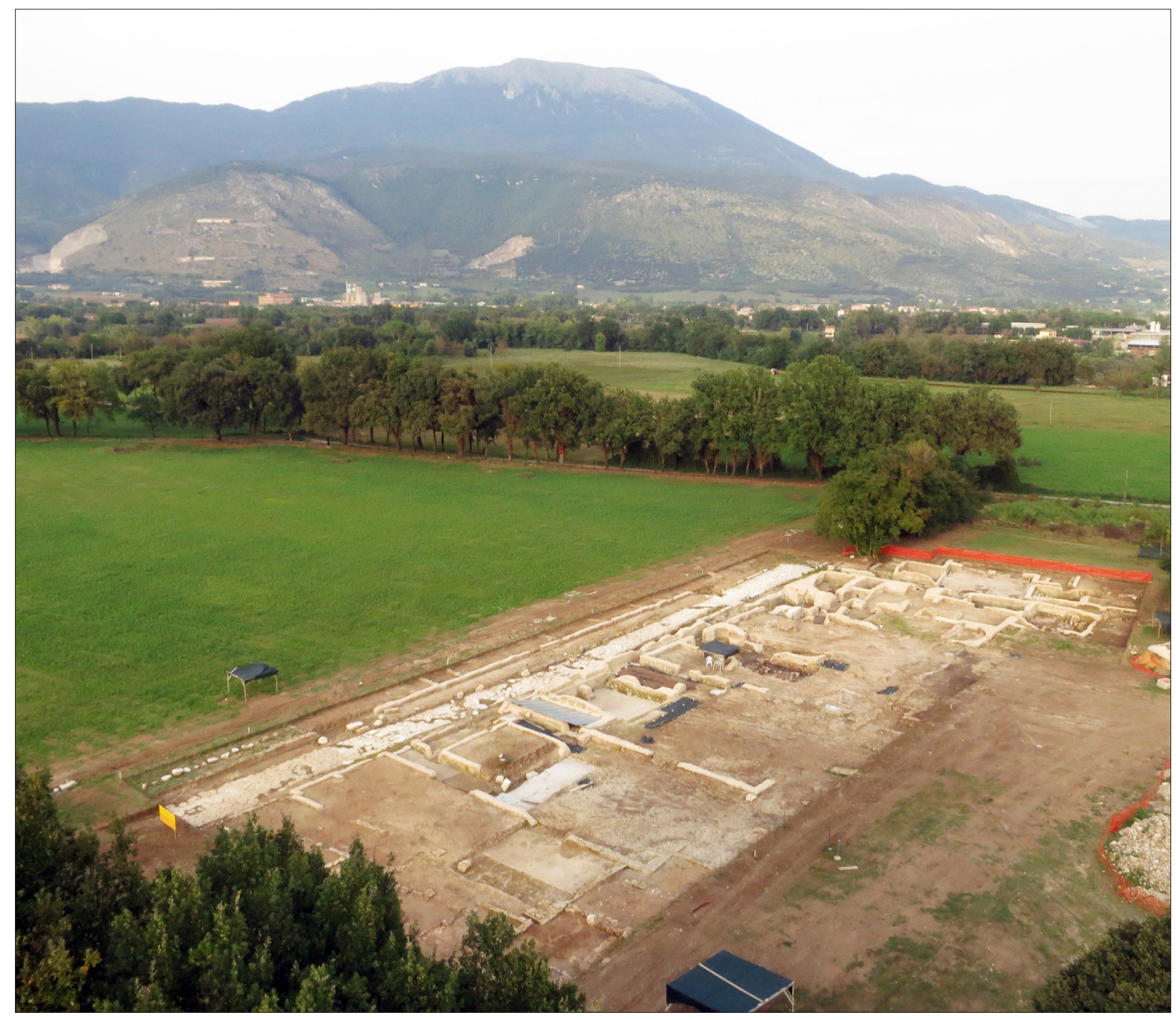

Fig. 2. Aquinum, Terme Centrali. Veduta aerea prospettica dell'area di scavo (foto G. Murro).

e da riformulazioni edilizie sul fronte orientale. Stando a quanto si può attualmente vedere, il complesso sembra essere costituito da un imponente blocco centrale, dove si sviluppano gli ambienti caldi e dove sono posizionati i praefurnia, e da due ali laterali per gli ambienti freddi le latrinae e gli ambienti di servizio a margine. Gli ambienti riscaldati risultano localizzati nel settore meridionale e centrale dell'area di scavo (Albiero et al, 2014: 1-20), ed occupano una superficie di oltre $400 \mathrm{mq}$. e la campagna di scavo 2014 ha messo in evidenza

almeno due ulteriori vani, pertinenti ad un calidarium e al laconicum, dalla caratteristica forma circolare. Anche questi, come del resto tutti gli altri ambienti riscaldati finora rinvenuti, presentano tracce cospicue delle suspensurae e scarsi resti del pavimento e dei tubuli fittili, presenti in gran quantità negli strati di crollo.

Un ulteriore gruppo di ambienti è posto nella parte occidentale del complesso edilizio. Sono stati scoperti due vani mosaicati, cui si affianca la latrina (fig. 5). Questa, di forma rettangolare, presenta un mosaico bicromo raffigurante una scena in ambiente nilotico con symplegma erotico al centro. La raffigurazione, di buona tecnica esecutiva, trova confronti soprattutto con casi ostiensi 


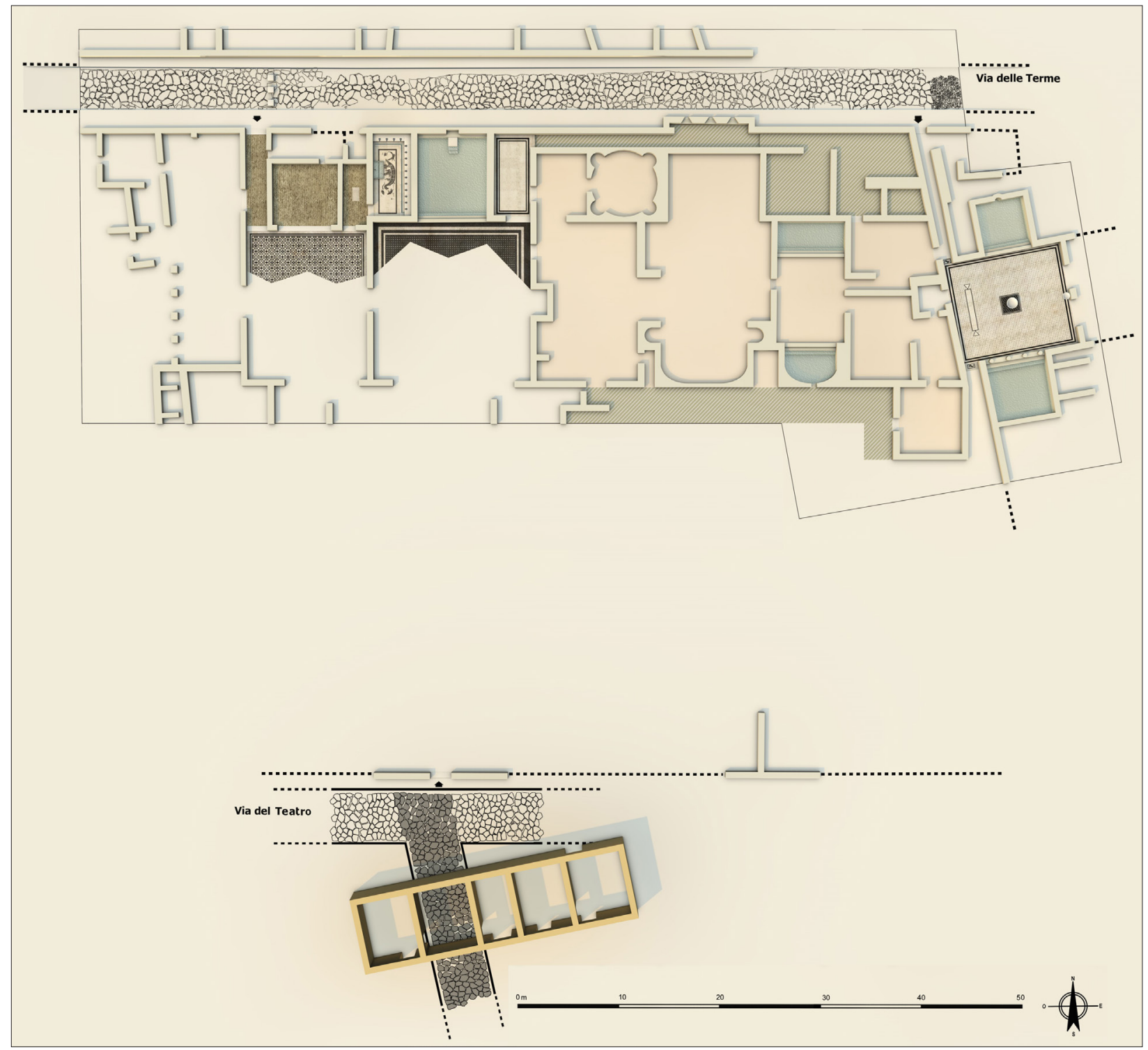

Fig. 3. Aquinum. La parte scavata dell'edificio termale e gli ambienti portati alla luce tra i due decumani paralleli alla via Latina (elaborazione grafica I. Ferrari).

e potrebbe essere datato intorno alla metà del II sec. d.C. Lo stato di conservazione della latrina permette di comprenderne con estrema facilità il funzionamento in tutte le parti caratterizzanti.

Il complesso termale si colloca cronologicamente tra la fine del I sec. a.C. e gli inizi del I sec. d.C., con una fase importante databile attraverso alcuni mosaici in età giulio-claudia. In un momen- to successivo, identificabile intorno alla metà del II sec. d.C., alcune parti del monumento subirono delle modifiche, in alcuni casi forse connesse a esigenze di carattere strutturale, come si può dedurre dal raddoppio dello spessore delle murature in alcuni punti.

Nel corso del IV sec. d.C., l'edificio inizia ad essere interessato in molte parti da un'attività di spoliazione che si intensifica soprattutto nel secolo successivo, indirizzata principalmente all'asportazione dei marmi e delle condutture in piombo. La mancanza di manufatti diagnostici ascrivibili al $\mathrm{V}$ sec. d.C., fanno pensare che il complesso fosse già in fase di totale disfacimento. L'unico settore che 


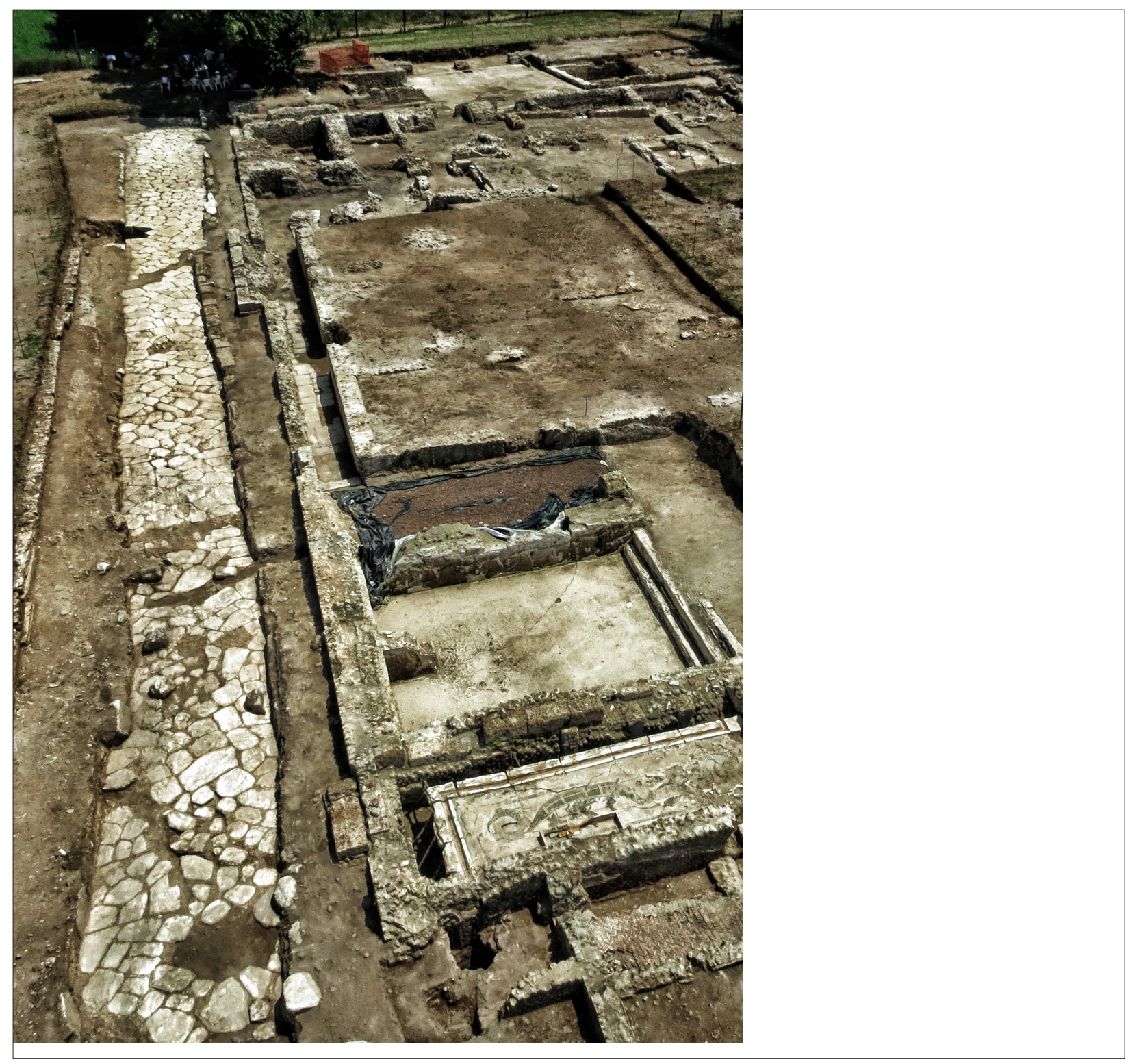

Fig. 4. Aquinum. Alcuni degli ambienti del complesso termale che si affacciano su via delle Terme (foto G. Murro).

presenta in questo periodo una continuità notevole nella frequentazione è il decumano a nord del complesso termale, che ha restituito abbondante materiale numismatico inquadrabile proprio in questa fase. Nel corso del VI e VII secolo parti del complesso vengono utilizzate come aree cimiteriali, verosimilmente a carattere familiare, in un tessuto urbano ormai ruralizzato. Le tombe, a fossa o più comunemente a cassone litico vengono ricavate di- rettamente negli strati di crollo e addossate ai muri. L'orizzonte cronologico delle stesse, ricavabile dai pochissimi dati materiali e dalle peculiarità costruttive delle sepolture, è inquadrabile tra metà VI/VII sec. d.C., dunque in un momento in cui che le fonti collocano l'arrivo dei Longobardi nella zona.

Allo stato attuale delle ricerche sembra che in alcune delle porzioni indagate, l'utilizzo dell'edificio si interrompa proprio in questo momento. In limitatissime parti sono state riconosciute attività riconducibili a funzioni di tipo abitativo, indiziate da focolari e fosse, databili al XIII-XIV sec. come evidenziato dai reperti ceramici recuperati. 


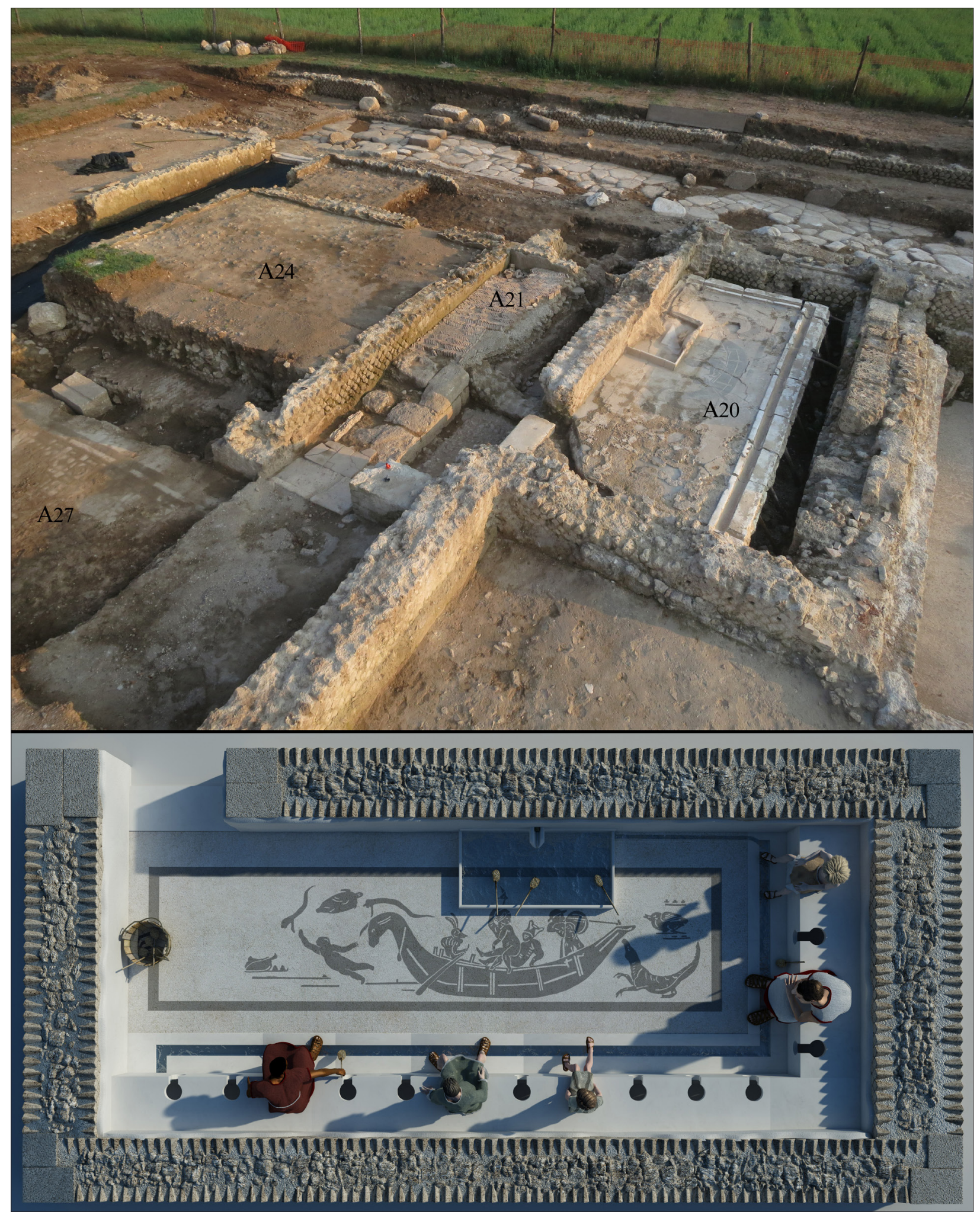

Fig. 5. Aquinum. Veduta aerea prospettica della latrina con il mosaico nilotico (foto G. Murro) e ricostruzione 3D, vista zenitale (elaborazione grafica I. Ferrari). 


\section{La valorizzazione delle conoscenze}

L'auspicio è che questi interventi all'interno dell'Area Archeologica di Aquinum potranno portare benefici in tutte le direzioni, consentendo un arricchimento del patrimonio di questa importante città romana nel Lazio meridionale, attraverso la messa in sicurezza di tutta l'area, l'allestimento in tempi ragionevoli di uno spazio specifico dedicato alle Terme presso i rinnovati locali del Casale Pascale e l'istituzione di un percorso di visita degli scavi, sicuro e adeguatamente attrezzato. Tutto nel rispetto delle diverse competenze e prerogative istituzionali, ai fini della conoscenza, della tutela e della valorizzazione dell'Area Archeologica aquinate e del ricco patrimonio che essa ancora custodisce.

In relazione alla possibilità di impiegare le strutture del Casale Pascale, recentemente restaurate come spazio espositivo relativo al sito e agli scavi nell'area centrale della colonia di Aquinum, si sta sviluppando un innovativo progetto di allestimento, che si propone di andare al di là della tradizionale musealizzazione dei reperti provenienti dallo scavo e prospetta uno spazio innovativo dove la rarefatta presenza degli oggetti più rappresentativi portati alla luce (statue, busti, rilievi architettonici), evochi la più ampia gamma di contenuti possibili. L'obiettivo principale è comunicare l'importanza del sito in maniera efficace, adatta a superare le differenze di età, cultura e interessi proprie di un pubblico ampio. L'interazione dello spazio espositivo col sito circostante sarà coinvolgente e raggiungibile tramite una serie di visualizzazioni interattive che offriranno finestre sul passato, mostrando l'aspetto originario di strutture e reperti, sfruttando le potenzialità delle ricostruzioni tridimensionali attraverso specifiche elaborazioni che in accoglienza permetteranno una immediata contestualizzazione dell'area all'interno della quale ci si trova tramite un modello plastico multimediale/interattivo e finestre virtuali sulle terme antiche, quindi un'esperienza immersiva tramite proiezioni interattive e $3 \mathrm{D}$ sulle pareti della stanza ed infine la possibilità di approfondimento post-visita con storytelling grazie alla narrazione di antichi personaggi storici aquinati (Giovenale, Cicerone, ecc.).

Mi auguro che lo sforzo di conoscenza che si sta cercando di portare avanti, possa costituire da volano e possa dare l'energia e le giuste motivazioni per intrecciare competenze e consapevolezze volte a rinnovare in maniera concreta il sistema della tutela in una strategia della valorizzazione: valorizzazione non solo dei beni ma anche delle conoscenze, che permettono non solo di arricchire questo Patrimonio, ma di studiarlo e capirlo nelle sue dinamiche storiche e topografiche.

\section{Bibliografia}

Albiero A, C Fernandez, V Petrucci, A Ugolini \& V Vitale 2014: Le terme centrali di Aquinum (Castrocielo, FR), Campagne di scavo 2009-2013, Folder. The Journal of Fasti Online, 316, 1-20.

Ceraudo G, 1999: Il contributo dell'aerofotogrammetria per la ricostruzione dell'impianto urbano di Aquinum, Terra dei Volsci, Annali, 2, 161-68.

Ceraudo G, 2004: Aquinum: la città romana, in Ceraudo $G$ (ed.), Ager Aquinas. Aerotopografia archeologica lungo la valle dell'antico Liris, Roma: 13-23. Caramanica, Marina di Minturno.

Ceraudo G (ed.) 2004: Ager Aquinas. Aereotopografia archeologica lungo la valle dell'antico Liris, Caramanica, Marina di Minturno.

Ceraudo G, 2012: Progetto “Ager Aquinas”. Indagini aerotopografiche finalizzate allo studio della Città Romana di Aquinum (Lazio, Italia), in Vermeulen F. Burgers GJ, Keay S \& Corsi C (ed.), Urban Landscape Survey in Italy and the Mediterranean: 94-103. Oxbow Books, Oxford

Ceraudo G, C Molle C \& D Nonnis 2013: L'iscrizione musiva delle Terme Centrali di Aquinum, Orizzonti. Rassegna di archeologia, 14, 101-109.

Ceraudo G \& G Murro 2014: Aquinum. Guida ai monumenti e all'area archeologica, Caludio Grenzi Editore, Foggia.

Giuliani CF, 1964: Aquino, in Quaderni dell'Istituto di Topografia Antica dell'Università di Roma, Saggi di fotointerpretazione archeologica, 1, Roma, 41-49. 
